\title{
Upper Extremity Selective Voluntary Motor Control in Children with Unilateral Cerebral Palsy and its association with Upper Extremity Function
}

\section{Ayse Yildiz, Ramazan Yildiz, Erkan Erol, Umut Apaydin, Muserrefe Nur Keles, Bulent Elbasan Department of Physical Therapy and Rehabilitation, Faculty of Health Sciences, Gazi University}

Background and Aims

Children with Cerebral Palsy (CP) reduced selective motor control (SMC) can prevent many common bimanual activities that require independent control of both hands. For this reason, it is aimed to define the relationship between upper extremity SMC and upper extremity function in children with unilateral CP.

\section{Method}

Twenty-six volunteer unilateral CP participated in the study between the ages of 6 and 18 years. Children's manual skills classified with MACS. To assess the quality of the upper extremity, the Quality of Upper Extremity Skill Test (QUEST) which assessed four areas including dissociated movement, grasp, weight bearing and protective extension, was used. Upper extremity functions were assessed using the Jebsen Taylor Hand Function Test (JTT). The Selektif Control of Upper Extremity Scale (SCUES) was used to evaluate the selective movements of the upper extremity. The SCUES evaluates the movement of the trunk during the upper limb movements and mirror movements via video recording.

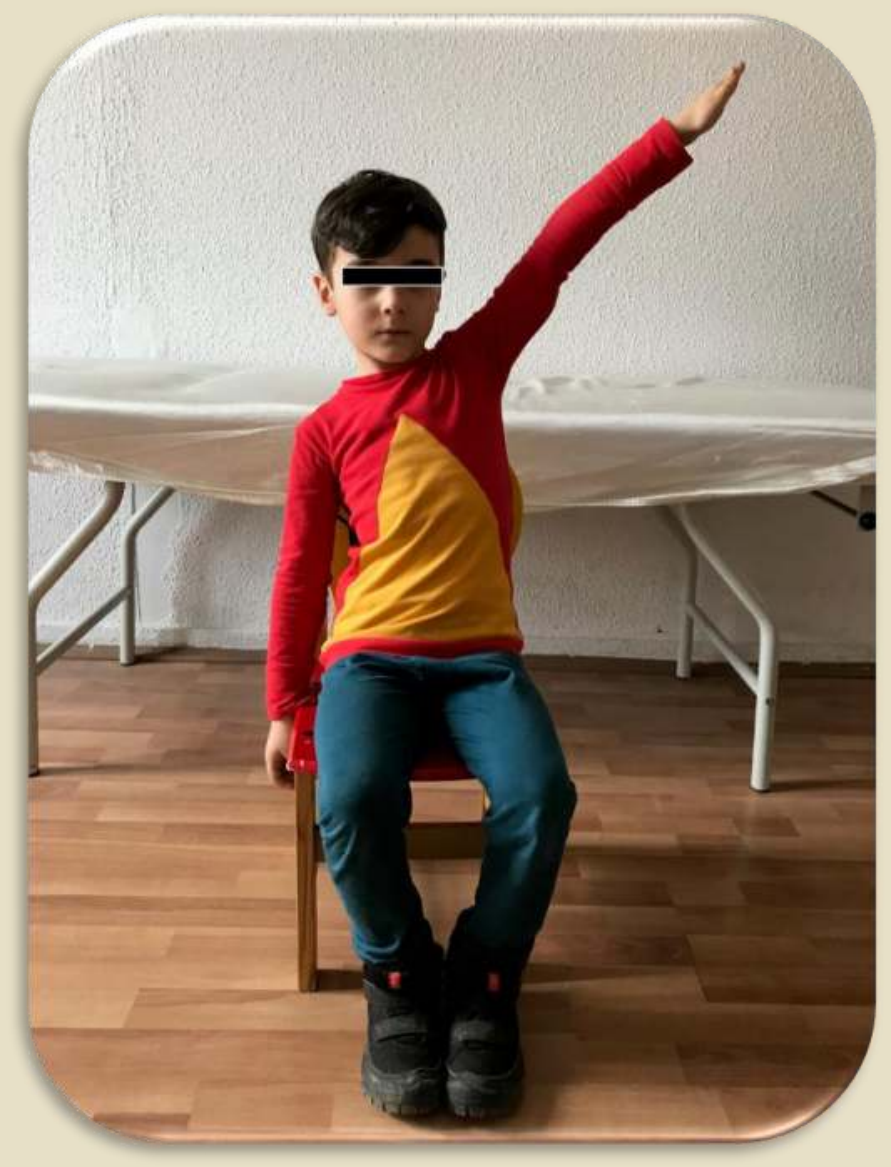

Results

Statistically significant decreases in SCUES scores from shoulder to fingers were found using the Page statistical test for trend $(\mathrm{p}<0.001)$. There was a very high positive correlation between SCUES total score and QUEST total score. There was a high negative correlation between the SCUES total score and the JTT total score (Table).

Table: Spearman's correlation coefficients between upper extremity functions and selective motor control

\begin{tabular}{|lcc|}
\hline \multicolumn{3}{|c}{ SCUES } \\
\hline JTT total score & 0.871 & $\mathrm{p}$ \\
\hline QUEST total score & 0.808 & $\mathrm{p}<0.001$ \\
\hline
\end{tabular}

\section{Conclusion}

In children with unilateral $\mathrm{CP}$, there was a relationship between upper extremity selective control and upper extremity functions. In children with hemiplegic cerebral palsy, the addition of SMC to the evaluation parameters has been found to be beneficial.

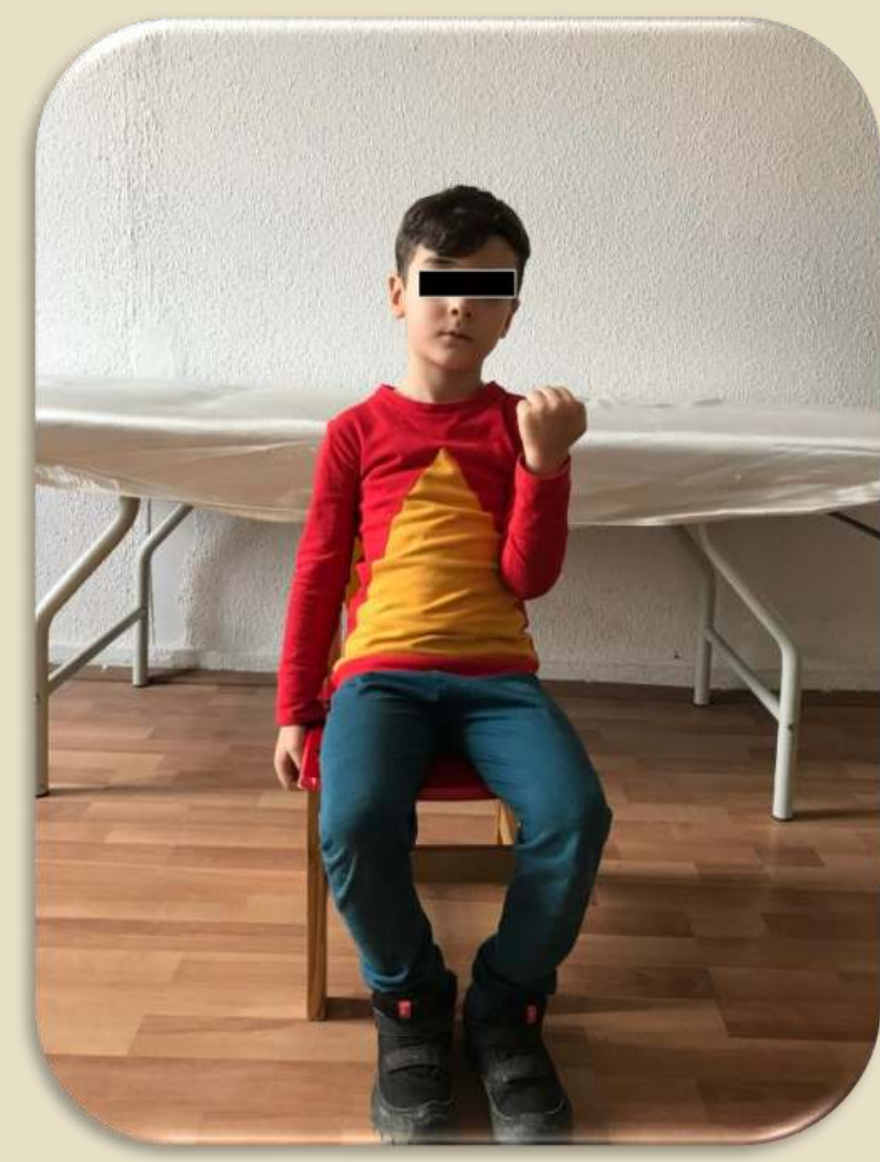

Adler, C., Berweck, S., Lidzba, K., Becher, T., \& Staudt, M. (2015). Mirror movements in unilateral spastic cerebral palsy: Specific negative impact on bimanual activities of daily living. european journal of paediatric neurology, 19(5), 504-509. 